\title{
Tribonacci numbers that are concatenations of two repdigits
}

\section{Mahadi Ddamulira ${ }^{1}$}

Received: 6 May 2020 / Accepted: 4 September 2020 / Published online: 15 September 2020

(c) The Author(s) 2020

\section{Abstract}

Let $\left(T_{n}\right)_{n \geq 0}$ be the sequence of Tribonacci numbers defined by $T_{0}=0, T_{1}=T_{2}=1$, and $T_{n+3}=T_{n+2}+T_{n+1}+T_{n}$ for all $n \geq 0$. In this note, we use of lower bounds for linear forms in logarithms of algebraic numbers and the Baker-Davenport reduction procedure to find all Tribonacci numbers that are concatenations of two repdigits.

Keywords Tribonacci number $\cdot$ repdigit $\cdot$ linear form in logarithms $\cdot$ reduction method Mathematics Subject Classification Primary 11B39 $\cdot$ 11D45 - Secondary 11D61 · 11J86

\section{Introduction}

A repdigit is a positive integer $R$ that has only one distinct digit when written in its decimal expansion. That is, $R$ is of the form

$$
R=\underbrace{\overline{d \cdots d}}_{\text {ltimes }}=d\left(\frac{10^{\ell}-1}{9}\right),
$$

for some positive integers $d, \ell$ with $\ell \geq 1$ and $0 \leq d \leq 9$. The sequence of repdigits is sequence $A 010785$ on the On-Line Encyclopedia of Integer Sequences (OEIS) [8].

Consider the sequence $\left(T_{n}\right)_{n \geq 0}$ of Tribonacci numbers given by

$$
T_{0}=0, \quad T_{1}=1, \quad T_{2}=1, \quad \text { and } \quad T_{n+3}=T_{n+2}+T_{n+1}+T_{n} \text { for all } n \geq 0 .
$$

The sequence of Tribonacci numbers is sequence $A 000073$ on the OEIS. The first few terms of this sequence are given by

$$
\left(T_{n}\right)_{n \geq 0}=\{0,1,1,2,4,7,13,24,44,81,149,274,504,927,1705,3136, \cdots\} .
$$

Mahadi Ddamulira

mddamulira@tugraz.at; mahadi@aims.edu.gh

1 Institute of Analysis and Number Theory, University of Technology, Kopernikusgasse 24/II, 8010 Graz, Austria 


\section{Main result}

In this paper, we study the problem of finding all Tribonacci numbers that are concatenations of two repdigits. More precisely, we completely solve the Diophantine equation

$$
T_{n}=\underbrace{\overline{d_{1} \cdots d_{1}} \underbrace{d_{2} \cdots d_{2}}_{\ell_{2} \text { times }}}_{\ell_{1} \text { times }}=d_{1}\left(\frac{10^{\ell_{q}}-1}{9}\right) \cdot 10^{\ell_{2}}+d_{2}\left(\frac{10^{\ell_{2}}-1}{9}\right),
$$

in non-negative integers $\left(n, d_{1}, d_{2}, \ell_{1}, \ell_{2}\right)$ with $n \geq 0, \ell_{1} \geq \ell_{2} \geq 1$, and $d_{1}, d_{2} \in$ $\{0,1, \ldots, 9\}, d_{1}>0$.

Our main result is the following.

Theorem 2.1 The only Tribonacci numbers that are concatenations of two repdigits are

$$
T_{n} \in\{13,24,44,81\} .
$$

Our method of proof involves the application of Baker's theory for linear forms in logarithms of algebraic numbers, and the Baker-Davenport reduction procedure. Computations are done with the help of a computer program in Mathematica.

Let $\left(F_{n}\right)_{n \geq 0}$ be the sequence of Fibonacci numbers given by $F_{0}=0, F_{1}=1$, and $F_{n+2}=F_{n+1}+F_{n}$ for all $n \geq 0$, and $\left(B_{n}\right)_{n \geq 0}$ be the sequence of balancing numbers given by $B_{0}=0, B_{1}=1$, and $B_{n+2}=6 B_{n+1}-B_{n}$ for all $n \geq 0$. This paper is inspired by the results of Alahmadi et al. [1], in which they show that the only Fibonacci numbers that are concatenations of two repdigits are $F_{n} \in\{13,21,34,55,89,144,233,377\}$, and Rayaguru and Panda [10], who showed that $B_{n} \in\{35\}$ is the only balancing number that can be written as a concatenation of two repdigits. Other related interesting results in this direction include: the result of Bravo and Luca [3], the result of Trojovský [11], the result of Qu and Zeng [9], and the result of Boussayoud et al. [2].

\section{Preliminary results}

\subsection{The Tribonacci sequence}

Here, we recall some important properties of the Tribonacci sequence $\left\{T_{n}\right\}_{n \geq 0}$. The characteristic equation

$$
\Psi(x):=x^{3}-x^{2}-x-1=0,
$$

has roots $\alpha, \beta, \gamma=\bar{\beta}$, where

$$
\alpha=\frac{1+\left(r_{1}+r_{2}\right)}{3}, \quad \beta=\frac{2-\left(r_{1}+r_{2}\right)+\sqrt{-3}\left(r_{1}-r_{2}\right)}{6},
$$

and

$$
r_{1}=\sqrt[3]{19+3 \sqrt{33}} \text { and } r_{2}=\sqrt[3]{19-3 \sqrt{33}}
$$

Further, the Binet formula for the general terms of the Tribonacci sequence is given by

$$
T_{n}=a \alpha^{n}+b \beta^{n}+c \gamma^{n} \quad \text { for all } n \geq 0,
$$

where

$$
a=\frac{1}{(\alpha-\beta)(\alpha-\gamma)}, \quad b=\frac{1}{(\beta-\alpha)(\beta-\gamma)}, \quad c=\frac{1}{(\gamma-\alpha)(\gamma-\beta)}=\bar{b} .
$$


Furthermore,

$$
a=\frac{\alpha}{\alpha^{2}+2 \alpha+3},
$$

and the minimal polynomial of $a$ over the integers is given by

$$
44 x^{3}+4 x-1
$$

has zeros $a, b, c$ with $\max \{|a|,|b|,|c|\}<1$. Numerically, the following estimates hold:

$$
\begin{aligned}
1.83 & <\alpha<1.84 \\
0.73<|\beta| & =|\gamma|=\alpha^{-\frac{1}{2}}<0.74 \\
0.18 & <a<0.19 \\
0.35 & <|b|=|c|<0.36
\end{aligned}
$$

From (3.1), (3.2), and (3.5), it is easy to see that the contribution the complex conjugate roots $\beta$ and $\gamma$, to the right-hand side of (3.3), is very small. In particular, setting

$$
e(n):=T_{n}-a \alpha^{n}=b \beta^{n}+c \gamma^{n} \quad \text { then } \quad|e(n)|<\frac{1}{\alpha^{n / 2}},
$$

holds for all $n \geq 1$. The proof of the last inequality in (3.6) follows from the fact that $|\beta|=|\gamma|=\alpha^{-\frac{1}{2}}$ and $|b|=|c|<0.36$ (by 3.5). That is, for any $n \geq 1$,

$$
|e(n)|=\left|b \beta^{n}+c \gamma^{n}\right| \leq|b||\beta|^{n}+|c||\gamma|^{n}=|b| \alpha^{-\frac{n}{2}}+|c| \alpha^{-\frac{n}{2}}<2 \cdot 0.36 \cdot \alpha^{-\frac{n}{2}}<\frac{1}{\alpha^{n / 2}} .
$$

Furthermore, by induction, one can prove that

$$
\alpha^{n-2} \leq T_{n} \leq \alpha^{n-1} \text { holds for all } n \geq 1
$$

Let $\mathbb{K}:=\mathbb{Q}(\alpha, \beta)$ be the splitting field of the polynomial $\Psi$ over $\mathbb{Q}$. Then, $[\mathbb{K}, \mathbb{Q}]=6$. Furthermore, $[\mathbb{Q}(\alpha): \mathbb{Q}]=3$. The Galois group of $\mathbb{K}$ over $\mathbb{Q}$ is given by

$$
\mathcal{G}:=\operatorname{Gal}(\mathbb{K} / \mathbb{Q}) \cong\{(1),(\alpha \beta),(\alpha \gamma),(\beta \gamma),(\alpha \beta \gamma),(\alpha \gamma \beta)\} \cong S_{3} .
$$

Thus, we identify the automorphisms of $\mathcal{G}$ with the permutations of the zeros of the polynomial $\Psi$. For example, the permutation $(\alpha \gamma)$ corresponds to the automorphism $\sigma: \alpha \rightarrow \gamma, \gamma \rightarrow$ $\alpha, \beta \rightarrow \beta$.

\subsection{Linear forms in logarithms}

Let $\eta$ be an algebraic number of degree $d$ with minimal primitive polynomial over the integers

$$
a_{0} x^{d}+a_{1} x^{d-1}+\cdots+a_{d}=a_{0} \prod_{i=1}^{d}\left(x-\eta^{(i)}\right),
$$

where the leading coefficient $a_{0}$ is positive and the $\eta^{(i)}$ 's are the conjugates of $\eta$. Then the logarithmic height of $\eta$ is given by

$$
h(\eta):=\frac{1}{d}\left(\log a_{0}+\sum_{i=1}^{d} \log \left(\max \left\{\left|\eta^{(i)}\right|, 1\right\}\right)\right) .
$$


In particular, if $\eta=p / q$ is a rational number with $\operatorname{gcd}(p, q)=1$ and $q>0$, then $h(\eta)=$ $\log \max \{|p|, q\}$. The following are some of the properties of the logarithmic height function $h(\cdot)$, which will be used in the next section of this paper without reference:

$$
\begin{aligned}
h\left(\eta_{1} \pm \eta_{2}\right) & \leq h\left(\eta_{1}\right)+h\left(\eta_{2}\right)+\log 2 ; \\
h\left(\eta_{1} \eta_{2}^{ \pm 1}\right) & \leq h\left(\eta_{1}\right)+h\left(\eta_{2}\right) ; \\
h\left(\eta^{s}\right) & =|s| h(\eta) \quad(s \in \mathbb{Z}) .
\end{aligned}
$$

We recall the result of Bugeaud et al. ([4], Theorem 9.4), which is a modified version of the result of Matveev [7], which is one of our main tools in this paper.

Theorem 3.1 Let $\eta_{1}, \cdots, \eta_{t}$ be positive real algebraic numbers in a real algebraic number field $\mathbb{K} \subset \mathbb{R}$ of degree $D, b_{1}, \cdots, b_{t}$ be nonzero integers, and assume that

$$
\Lambda:=\eta_{1}^{b_{1}} \cdots \eta_{t}^{b_{t}}-1 \neq 0
$$

Then,

$$
\log |\Lambda|>-1.4 \cdot 30^{t+3} \cdot t^{4.5} \cdot D^{2}(1+\log D)(1+\log B) A_{1} \cdots A_{t}
$$

where

$$
B \geq \max \left\{\left|b_{1}\right|, \cdots,\left|b_{t}\right|\right\}
$$

and

$$
A_{i} \geq \max \left\{D h\left(\eta_{i}\right),\left|\log \eta_{i}\right|, 0.16\right\}, \quad \text { for all } \quad i=1, \cdots, t
$$

\subsection{Reduction procedure}

During the calculations, we get upper bounds on our variables which are too large, thus we need to reduce them. To do so, we use some result from the theory of continued fractions. For a nonhomogeneous linear form in two integer variables, we use a slight variation of a result due to Dujella and Pethő ([5], Lemma 5a). For a real number $X$, we write $\|X\|:=$ $\min \{|X-n|: n \in \mathbb{Z}\}$ for the distance from $X$ to the nearest integer.

Lemma 3.1 Let $M$ be a positive integer, $\frac{p}{q}$ be a convergent of the continued fraction expansion of the irrational number $\tau$ such that $q>6 M$, and $A, B, \mu$ be some real numbers with $A>0$ and $B>1$. Furthermore, let $\varepsilon:=\|\mu q\|-M\|\tau q\|$. If $\varepsilon>0$, then there is no solution to the inequality

$$
0<|u \tau-v+\mu|<A B^{-w},
$$

in positive integers $u, v$, and $w$ with

$$
u \leq M \quad \text { and } w \geq \frac{\log (A q / \varepsilon)}{\log B} .
$$

The following Lemma is also useful. It is due to Gúzman Sánchez and Luca ([6], Lemma 7).

Lemma 3.2 If $r \geq 1, H>\left(4 r^{2}\right)^{r}$, and $H>L /(\log L)^{r}$, then

$$
L<2^{r} H(\log H)^{r} .
$$




\section{The proof of Theorem 2.1}

\subsection{The small ranges}

With the help of Mathematica, we checked all the solutions to the Diophantine equation (2.1) in the ranges $0 \leq d_{2}<d_{1} \leq 9$ and $1 \leq \ell_{2} \leq \ell_{1} \leq n \leq 200$ and found only the solutions stated in Theorem 2.1. From now on we assume that $n>200$.

\subsection{The initial bound on $n$}

We rewrite (2.1) as

$$
\begin{aligned}
T_{n} & =\underbrace{\overline{d_{1} \cdots d_{1}} \underbrace{d_{2} \cdots d_{2}}_{\ell_{2}}}_{\ell_{1} \text { times }} \\
& =\underbrace{\overline{d_{1} \cdots d_{1}}}_{\ell_{1} \text { times }} \cdot 10^{\ell_{2}}+\underbrace{\overline{d_{2} \cdots d_{2}}}_{\ell_{2} \text { times }} \\
& =d_{1}\left(\frac{10^{\ell_{1}}-1}{9}\right) \cdot 10^{\ell_{2}}+d_{2}\left(\frac{10^{\ell_{2}}-1}{9}\right) \\
& =\frac{1}{9}\left(d_{1} \cdot 10^{\ell_{1}+\ell_{2}}-\left(d_{1}-d_{2}\right) \cdot 10^{\ell_{2}}-d_{2}\right) .
\end{aligned}
$$

Thus,

$$
T_{n}=\frac{1}{9}\left(d_{1} \cdot 10^{\ell_{1}+\ell_{2}}-\left(d_{1}-d_{2}\right) \cdot 10^{\ell_{2}}-d_{2}\right) .
$$

We prove the following lemma, which gives a relation on the size of $n$ versus $\ell_{1}+\ell_{2}$.

Lemma 4.1 All solutions of the Diophantine equation (4.1) satisfy

$$
\left(\ell_{1}+\ell_{2}\right) \log 10-2<n \log \alpha<\left(\ell_{1}+\ell_{2}\right) \log 10+2 .
$$

Proof The proof follows easily from (3.7). One can see that

$$
\alpha^{n-2} \leq T_{n}<10^{\ell_{1}+\ell_{2}} .
$$

Taking the logarithm on both sides, we get that

$$
(n-2) \log \alpha<\left(\ell_{1}+\ell_{2}\right) \log 10,
$$

which leads to

$$
n \log \alpha<\left(\ell_{1}+\ell_{2}\right) \log 10+2 \log \alpha<\left(\ell_{1}+\ell_{2}\right) \log 10+2 .
$$

For the lower bound, we have that

$$
10^{\ell_{1}+\ell_{2}-1}<T_{n} \leq \alpha^{n-1} .
$$

Taking the logarithm on both sides, we get that

$$
\left(\ell_{1}+\ell_{2}-1\right) \log 10<(n-1) \log \alpha,
$$

which leads to

$$
\left(\ell_{1}+\ell_{2}\right) \log 10-2<\left(\ell_{1}+\ell_{2}-1\right) \log 10+\log \alpha<n \log \alpha .
$$

Comparing (4.2) and (4.3) gives the result in the lemma. 
Next, we examine (4.1) in two different steps.

Step 1 Substituting (3.3) in (4.1), we get that

$$
a \alpha^{n}+b \beta^{n}+c \gamma^{n}=\frac{1}{9}\left(d_{1} \cdot 10^{\ell_{1}+\ell_{2}}-\left(d_{1}-d_{2}\right) \cdot 10^{\ell_{2}}-d_{2}\right) .
$$

By (3.6), this is equivalent to

$$
9 a \alpha^{n}-d_{1} \cdot 10^{\ell_{1}+\ell_{2}}=-9 e(n)-\left(d_{1}-d_{2}\right) \cdot 10^{\ell_{2}}-d_{2},
$$

from which we deduce that

$$
\begin{aligned}
\left|9 a \alpha^{n}-d_{1} \cdot 10^{\ell_{1}+\ell_{2}}\right| & =\left|9 e(n)+\left(d_{1}-d_{2}\right) \cdot 10^{\ell_{2}}+d_{2}\right| \\
& \leq 9 \alpha^{-n / 2}+9 \cdot 10^{\ell_{2}}+9 \\
& <28 \cdot 10^{\ell_{2}} .
\end{aligned}
$$

Thus, dividing both sides by $d_{1} \cdot 10^{\ell_{1}+\ell_{2}}$ we get that

$$
\left|\left(\frac{9 a}{d_{1}}\right) \cdot \alpha^{n} \cdot 10^{-\ell_{1}-\ell_{2}}-1\right|<\frac{28 \cdot 10^{\ell_{2}}}{d_{1} \cdot 10^{\ell_{1}+\ell_{2}}}<\frac{28}{10^{\ell_{1}}} .
$$

Put

$$
\Lambda_{1}:=\left(\frac{9 a}{d_{1}}\right) \cdot \alpha^{n} \cdot 10^{-\ell_{1}-\ell_{2}}-1 .
$$

Next, we apply Theorem 3.1 on (4.5). First, we need to check that $\Lambda_{1} \neq 0$. If it were, then we would get that

$$
a \alpha^{n}=\frac{d_{1}}{9} \cdot 10^{\ell_{1}+\ell_{2}} .
$$

Now, we apply the automorphism $\sigma$ of the Galois group $\mathcal{G}$ on both sides and take absolute values as follows.

$$
\left|\frac{d_{1}}{9} \cdot 10^{\ell_{1}+\ell_{2}}\right|=\left|\sigma\left(a \alpha^{n}\right)\right|=\left|c \gamma^{n}\right|<1,
$$

which is false. Thus, $\Lambda_{1} \neq 0$. So, we apply Theorem 3.1 on (4.5) with the data:

$$
t:=3, \quad \eta_{1}:=\frac{9 a}{d_{1}}, \quad \eta_{2}:=\alpha, \quad \eta_{3}:=10, \quad b_{1}:=1, \quad b_{2}:=n, \quad b_{3}:=-\ell_{1}-\ell_{2} .
$$

By Lemma 4.1, we have that $\ell_{1}+\ell_{2}<n$. Therefore, we can take $B:=n$. Observe that $\mathbb{K}:=\mathbb{Q}\left(\eta_{1}, \eta_{2}, \eta_{3}\right)=\mathbb{Q}(\alpha)$, since $a=\alpha /\left(\alpha^{2}+2 \alpha+3\right)$, so $D:=3$. We have

$$
h\left(\eta_{1}\right)=h\left(9 a / d_{1}\right) \leq h(9)+h(a)+h\left(d_{1}\right) \leq \log 9+\frac{1}{3} \log 44+\log 9 \leq 5.66 .
$$

Furthermore, $h\left(\eta_{2}\right)=h(\alpha)=(1 / 3) \log \alpha$ and $h\left(\eta_{3}\right)=h(10)=\log 10$. Thus, we can take

$$
A_{1}:=16.98, \quad A_{2}:=\log \alpha, \quad \text { and } \quad A_{3}:=3 \log 10 .
$$

Theorem 3.1 tells us that

$$
\begin{aligned}
\log \left|\Lambda_{1}\right| & >-1.4 \cdot 30^{6} \cdot 3^{4.5} \cdot 3^{2}(1+\log 3)(1+\log n)(16.98)(\log \alpha)(3 \log 10) \\
& >-1.94 \cdot 10^{14}(1+\log n) .
\end{aligned}
$$

Comparing the above inequality with (4.4) gives

$$
\ell_{1} \log 10-\log 28<1.94 \cdot 10^{14}(1+\log n),
$$


leading to

$$
\ell_{1} \log 10<1.96 \cdot 10^{14}(1+\log n) .
$$

Step 2 By (3.6), we rewrite (4.1) as

$$
9 a \alpha^{n}-\left(d_{1} \cdot 10^{\ell_{1}}-\left(d_{1}-d_{2}\right)\right) \cdot 10^{\ell_{2}}=-9 e(n)-d_{2},
$$

from which we deduce that

$$
\left|9 a \alpha^{n}-\left(d_{1} \cdot 10^{\ell_{1}}-\left(d_{1}-d_{2}\right)\right) \cdot 10^{\ell_{2}}\right|=\left|9 e(n)+d_{2}\right| \leq 9 \alpha^{-n / 2}+9<18 .
$$

Thus, dividing both sides by $9 a \alpha^{n}$ we get that

$$
\left|\left(\frac{d_{1} \cdot 10^{\ell_{1}}-\left(d_{1}-d_{2}\right)}{9 a}\right) \cdot \alpha^{-n} \cdot 10^{\ell_{2}}-1\right|<\frac{18}{9 a \alpha^{n}}<\frac{2}{\alpha^{n}} .
$$

Put

$$
\Lambda_{2}:=\left(\frac{d_{1} \cdot 10^{\ell_{1}}-\left(d_{1}-d_{2}\right)}{9 a}\right) \cdot \alpha^{-n} \cdot 10^{\ell_{2}}-1 .
$$

Next, we apply Theorem 3.1 on (4.8). First, we need to check that $\Lambda_{2} \neq 0$. If not, then we would get that

$$
a \alpha^{n}=\left(\frac{d_{1} \cdot 10^{\ell_{1}}-\left(d_{1}-d_{2}\right)}{9}\right) \cdot 10^{\ell_{2}} .
$$

As before, we apply the automorphism $\sigma$ of the Galois group $\mathcal{G}$ on both sides and take absolute values as follows.

$$
\left|\left(\frac{d_{1} \cdot 10^{\ell_{1}}-\left(d_{1}-d_{2}\right)}{9}\right) \cdot 10^{\ell_{2}}\right|=\left|\sigma\left(a \alpha^{n}\right)\right|=\left|c \gamma^{n}\right|<1,
$$

which is false. Thus, $\Lambda_{2} \neq 0$. So, we apply Theorem 3.1 on (4.8) with the data:

$$
\begin{aligned}
t & :=3, \quad \eta_{1}:=\frac{d_{1} \cdot 10^{\ell_{1}}-\left(d_{1}-d_{2}\right)}{9 a}, \quad \eta_{2}:=\alpha, \quad \eta_{3}:=10, \quad b_{1}:=1, \\
& b_{2}:=-n, \quad b_{3}:=\ell_{2} .
\end{aligned}
$$

As before, we have that $\ell_{2}<n$. Thus, we can take $B:=n$. Similarly, $\mathbb{Q}\left(\eta_{1}, \eta_{2}, \eta_{3}\right)=\mathbb{Q}(\alpha)$, so we take $D:=3$. Furthermore, we have

$$
\begin{aligned}
h\left(\eta_{1}\right) & =h\left(\frac{d_{1} \cdot 10^{\ell_{1}}-\left(d_{1}-d_{2}\right)}{9 a}\right) \\
& \leq h\left(d_{1} \cdot 10^{\ell_{1}}-\left(d_{1}-d_{2}\right)\right)+h(9 a) \\
& \leq h\left(d_{1} \cdot 10^{\ell_{1}}\right)+h\left(d_{1}-d_{2}\right)+h(9)+h(a)+\log 2 \\
& \leq h\left(d_{1}\right)+\ell_{1} h(10)+h\left(d_{1}\right)+h\left(d_{2}\right)+h(9)+h(a)+2 \log 2 \\
& \leq \ell_{1} \log 10+4 \log 9+\frac{1}{3} \log 44+2 \log 2 \\
& \leq 1.96 \cdot 10^{14}(1+\log n)+4 \log 9+\frac{1}{3} \log 44+2 \log 2 \quad \text { (by (4.6)) } \\
& <1.98 \cdot 10^{14}(1+\log n) .
\end{aligned}
$$

Thus, we can take

$$
A_{1}:=5.94 \cdot 10^{14}(1+\log n), \quad A_{2}:=\log \alpha, \quad \text { and } \quad A_{3}:=3 \log 10 .
$$


Theorem 3.1 tells us that

$$
\begin{aligned}
\log \left|\Lambda_{2}\right| & >-1.4 \cdot 30^{6} \cdot 3^{4.5} \cdot 3^{2}(1+\log 3)(1+\log n)\left(5.94 \cdot 10^{14}(1+\log n)\right)(\log \alpha)(3 \log 10) \\
& >-6.77 \cdot 10^{27}(1+\log n)^{2} .
\end{aligned}
$$

Comparing the above inequality with (4.7) gives,

$$
n \log \alpha-\log 2<6.77 \cdot 10^{27}(1+\log n)^{2},
$$

which is equivalent to

$$
n<2.24 \cdot 10^{28}(\log n)^{2} .
$$

Applying Lemma 3.2 on (4.9) with the data $r=2, H:=2.24 \cdot 10^{28}$, and $L:=n$, gives

$$
n<1.22 \cdot 10^{32} \text {. }
$$

Lemma 4.1 implies that

$$
\ell_{1}+\ell_{2}<3.24 \cdot 10^{31}
$$

We have just proved the following lemma.

Lemma 4.2 All solutions to the Diophantine equation (4.1) satisfy

$$
\ell_{1}+\ell_{2}<3.24 \cdot 10^{31} \text { and } n<1.22 \cdot 10^{32} \text {. }
$$

\subsection{Reducing the bounds}

The bounds given in Lemma 4.2 are too large to carry out meaningful computation. Thus, we need to reduce them. To do so, we apply Lemma 3.1 as follows.

First, we return to (4.4) and put

$$
\Gamma_{1}:=\left(\ell_{1}+\ell_{2}\right) \log 10-n \log \alpha-\log \left(\frac{9 a}{d_{1}}\right) .
$$

The inequality (4.4) can be rewritten as

$$
\left|e^{-\Gamma_{1}}-1\right|<\frac{28}{10^{\ell_{1}}} .
$$

Assume that $\ell_{1} \geq 2$, then the right-hand side in the above inequality is at most $7 / 25<1 / 2$. The inequality $\left|e^{z}-1\right|<w$ for real values of $z$ and $w$ implies that $z<2 w$. Thus,

$$
\left|\Gamma_{1}\right|<\frac{56}{10^{\ell_{1}}}
$$

which implies that

$$
\left|\left(\ell_{1}+\ell_{2}\right) \log 10-n \log \alpha-\log \left(\frac{9 a}{d_{1}}\right)\right|<\frac{56}{10^{\ell_{1}}} .
$$

Dividing through by $\log \alpha$ gives

$$
\left|\left(\ell_{1}+\ell_{2}\right) \frac{\log 10}{\log \alpha}-n+\left(\frac{\log \left(d_{1} / 9 a\right)}{\log \alpha}\right)\right|<\frac{56}{10^{\ell_{1}} \log \alpha} .
$$

So, we apply Lemma 3.1 with the data:

$$
\tau:=\frac{\log 10}{\log \alpha}, \quad \mu\left(d_{1}\right):=\frac{\log \left(d_{1} / 9 a\right)}{\log \alpha}, \quad A:=\frac{56}{\log \alpha}, \quad B:=10, \quad 1 \leq d_{1} \leq 9 .
$$


Let $\tau=\left[a_{0} ; a_{1}, a_{2}, \cdots\right]=[3 ; 1,3,1,1,14,1,3,3,6,1,13,3,4,2,1,1,2,3,3,2, \cdots]$ be the continued fraction expansion of $\tau$. We choose $M:=10^{32}$ which is the upper bound on $\ell_{1}+\ell_{2}$. With the help of Mathematica, we find out that the convergent

$$
\frac{p}{q}=\frac{p_{62}}{q_{62}}=\frac{5067116767207083507605709005080661}{1341009632511071028566373818645201},
$$

is such that $q=q_{62}>6 M$. Furthermore, it yields $\varepsilon>0.0893601$, and therefore either

$$
\ell_{1} \leq \frac{\log ((56 / \log \alpha) q / \varepsilon)}{\log 10}<35,
$$

Thus, we have that $\ell_{1} \leq 35$.

For fixed $0 \leq d_{2}<d_{1} \leq 9$ and $1 \leq \ell_{1} \leq 35$, we return to (4.7) and put

$$
\Gamma_{2}:=\ell_{2} \log 10-n \log \alpha+\log \left(\frac{d_{1} \cdot 10^{\ell_{1}}-\left(d_{1}-d_{2}\right)}{9 a}\right) .
$$

From the inequality (4.7), we have that

$$
\left|e^{\Gamma_{2}}-1\right|<\frac{2}{\alpha^{n}}
$$

Since $n>200$, the right-hand side of the above inequality is less than $1 / 2$. Thus, the above inequality implies that

$$
\left|\Lambda_{1}\right|<\frac{4}{\alpha^{n}}
$$

which leads to

$$
\left|\ell_{2} \log 10-n \log \alpha+\log \left(\frac{d_{1} \cdot 10^{\ell_{1}}-\left(d_{1}-d_{2}\right)}{9 a}\right)\right|<\frac{4}{\alpha^{n}} .
$$

Dividing through by $\log \alpha$ gives,

$$
\left|\ell_{2}\left(\frac{\log 10}{\log \alpha}\right)-n+\frac{\log \left(\left(d_{1} \cdot 10^{\ell_{1}}-\left(d_{1}-d_{2}\right)\right) / 9 a\right)}{\log \alpha}\right|<\frac{4}{\alpha^{n} \log \alpha} .
$$

Again, we apply Lemma 3.1 with the data:

$$
\tau:=\frac{\log 10}{\log \alpha}, \quad \mu\left(d_{1}, d_{2}\right):=\frac{\log \left(\left(d_{1} \cdot 10^{\ell_{1}}-\left(d_{1}-d_{2}\right)\right) / 9 a\right)}{\log \alpha}, \quad A:=\frac{4}{\log \alpha}, \quad B:=\alpha .
$$

We take the same $\tau$ and its convergent $p / q=p_{62} / q_{62}$ as before. We choose $\ell_{2}<10^{32}:=M$. With the help of Mathematica, we get that $\varepsilon>0.0000798749$, and therefore

$$
n \leq \frac{\log ((4 / \log \alpha) q / \varepsilon)}{\log \alpha}<143 .
$$

Thus, we have that $n \leq 143$, contradicting the assumption that $n>200$. Hence, Theorem 2.1 is proved.

Acknowledgements The author thanks the anonymous referees and the editor for the careful reading of the manuscript, and the useful comments and suggestions that greatly improved the quality of presentation of the current paper.

Funding Open access funding provided by Austrian Science Fund (FWF). The author is supported by the Austrian Science Fund (FWF) projects: F05510-N26-Part of the special research program (SFB), "QuasiMonte Carlo Methods: Theory and Applications" and W1230-Doctoral Program Discrete Mathematics 
Open Access This article is licensed under a Creative Commons Attribution 4.0 International License, which permits use, sharing, adaptation, distribution and reproduction in any medium or format, as long as you give appropriate credit to the original author(s) and the source, provide a link to the Creative Commons licence, and indicate if changes were made. The images or other third party material in this article are included in the article's Creative Commons licence, unless indicated otherwise in a credit line to the material. If material is not included in the article's Creative Commons licence and your intended use is not permitted by statutory regulation or exceeds the permitted use, you will need to obtain permission directly from the copyright holder. To view a copy of this licence, visit http://creativecommons.org/licenses/by/4.0/.

\section{References}

1. Alahmadi, A., Altassan, A., Luca, F., Shoaib, H.: Fibonacci numbers which are concatenations of two repdigits. Quaest. Math. (2019). https://doi.org/10.2989/16073606.2019.1686439

2. Boussayoud, A., Boughaba, S., Kerada, M., Araci, S., Acikgoz, M.: Generating functions of binary products of $k$-Fibonacci and orthogonal polynomials. Rev. R. Acad. Cienc. Exactas Fís. Nat. Ser. A Mat. RACSAM 113(3), 2575-2586 (2019)

3. Bravo, J.J., Luca, F.: On a conjecture about repdigits in $k$-generalized Fibonacci sequences. Publ. Math. Debreceen 82(3-4), 623-639 (2013)

4. Bugeaud, Y., Mignotte, M., Siksek, S.: Classical Classical and modular approaches to exponential Diophantine equations I. Fibonacci and Lucas perfect powers. Ann. Math. 163(2), 969-1018 (2006)

5. Dujella, A., Pethő, A.: A generalization of a theorem of Baker and Davenport. Quart. J. Math. Oxf. Ser. 49(195), 291-306 (1998)

6. Gúzman Sánchez, S., Luca, F.: Linear combinations of factorials and $s$-units in a binary recurrence sequence. Ann. Math. Qué. 38(2), 169-188 (2014)

7. Matveev, E.M.: An explicit lower bound for a homogeneous rational linear form in the logarithms of algebraic numbers II. Izv. Ross. Akad. Nauk Ser. Math. 64(6), 125-180 (2000). in Russian: English translation in Izv. Math. 64(6), 1217-1269 (2000)

8. OEIS Foundation Inc.: The On-Line Encyclopedia of Integer Sequences (2020). https://oeis.org

9. Qu, Y., Zeng, J.: Lucas numbers which are concatenations of two repdigits. Mathematics 8(8), 1360 (2020). https://doi.org/10.3390/math8081360

10. Rayaguru, S.G., Panda, G.K.: Balancing numbers which are concatenations of two repdigits. Bol. Soc. Mat. Mex. (2020). https://doi.org/10.1007/s40590-020-00293-0

11. Trojovský, P.: Fibonacci numbers with a prescribed block of digits. Mathematics 8(4), 639 (2020). https:// doi.org/10.3390/math8040639

Publisher's Note Springer Nature remains neutral with regard to jurisdictional claims in published maps and institutional affiliations. 